\title{
Numeric modeling method usage for heat conductivity equations solution
}

\author{
Vladimir Loginov, Alla Stolyarova and Azad Parpiev \\ National Research Tomsk Polytechnic University, 30, Lenin Avenue, 634050 Tomsk, Russia
}

\begin{abstract}
The sweep method applicability for non-stationary one-dimensional differential heat conductivity equations solving is proved in this work. The sweep method applicability check is made by using exact analytical method and approximate analytical method. The confrontation carried out has shown that the obtained numeric results error isn't more $2 \%$.
\end{abstract}

\section{Introduction}

Heat-and-power engineering plays a great importance in our life. The steam heat, manufactured at power stations, as the main product is usefully transformed, for example into the mechanical or electrical energy. The different industry normal functioning depends directly on TPS (thermal power stations) and HPP (heat and power plants. That's why TPS and HPP equipment maintenance working has a great importance. At industry enterprises different preventive measures against superheating tubes damages are being developed based on the experimental data and thermal and hydraulic calculation results [1-3].

Nowadays a special attention is given to the starting and stopping processes influence, occurring in the boiler superheater metal. It is necessary to note that the superheater tubes service life is decreased by the complex non-stationary processes with the unsteady parameter values, the high thermal mechanical stresses, different elements elongation, the possible mechanical integrity contravention, the talent deformation occurrence, appearing during boiler starting and stopping processes.

And so nowadays the actual problem is the processes analysis, occurring in the tube metal at the intrastructural level by the non-stationary regimes influence, because overheating surfaces damage cause can explain only these processes.

\subsection{Short analysis of the concerned problem situation}

Nowadays there are many works [4-8] and others as to the superheater metal thermal mechanical changing properties investigation by both foreign and Russian authors at the intrastructural level, providing the external and internal factors. The authors of these works note that the II type crystalline microstresses and I type zonal microstresses are the main damage causes. These stresses result in the intercrystalline and transcrystalline corrosion. Crystal lattice thermal deformations, which are a I and II type phase changes consequence, are occured. Creep microfissures and pores small chains are created. It's necessary to note, that the boiler starting and stopping influence correct structured information at

\footnotetext{
${ }^{a}$ Corresponding author: azatparpiev@mail.ru
}

This is an Open Access article distributed under the terms of the Creative Commons Attribution License 4.0, which permits unrestricted use, distribution, and reproduction in any medium, provided the original work is properly cited. 
the all superheater temperature regime and thermal stresses isn't provide in these works. The operation non-stationary regimes temperature distributions are relative to radial and irrotational components and are known to change in different superheater elements because of the boilers operation practice and experimental data. Consequently the thermal stresses will be distributed differently depending on these temperature values. It's necessary to note that the thermal stresses change nature hasn't been well established. Different steel grades thermal mechanical properties of anomalous changes are especially observed at the temperature within the $650-720^{\circ} \mathrm{C}$ range, as noted by many authors. These anomalous changes nature hasn't been established. The phase changes, occurring at the intracrystalline level at this temperature range, are these changes cause, due to many authors hypothesizes. The data of different depositions availability types and their amount on the heating surface, the superheater tube temperature regime influence, aren't stated by the authors. It's one more of the shortcomings.

The tube metal remaining life forecasting method development, directly relating to the thermal stresses changes nature, is also one of the boilers superheaters investigation promising fields [9, 10]. Requiring an expensive equipment and significant financial expenditure usage, an experimental method is the main these works investigation way, as it was noted above. It's difficult to obtain the error-free information about the cylindrical tube wall radial and longitudinal components temperature distribution by using an experimental method. This aspect makes the thermal stresses determination process complicate. As to the analytical method, it's more complicated and impossible to solve similar problems in this way. Therefore numerical modeling usage for these purposes is more rational method. The results, obtained for a correct problem statement, are free-error.

Nowadays there are different numerical ways, such as: integration and differentiation methods. There are Runge-Kutt method, implicit schemes, shooting method, Galerkin method and the others [11]. Every method has its own advantages and disadvantages. The finite difference method is more simple and applicable to solve the above-listed problems, because there are the arithmetic operations of few quality for its efficiency and low sensitivity for errors calculating (absolute stability).

And so this work aim is the following. Let's calculating the known test problem, given in the work [12], for a sweep method applicability check to solve non-stationary one-dimensional differential heat conductivity equations. Let's analyze the given results confrontation with the results, obtained by means of an exact analytical method and an approximate analytical method usage, the algorithms of which is given in $[13,14]$. Basing on this analysis it's possible to come to conclusion that this numerical modeling method is applicable for a similar problems solution.

There are concerned tasks in this work, such as:

1. The physics-mathematical formulation for a superheater tube element non-stationary temperature regime.

2. The check of results validity, obtained during physic-mathematical model numerical computation.

\section{Physical problem statement}

The hollow cylinder, placed in the constant temperature medium, is the investigation object (Fig. 1). At the same time this object temperature is more than the medium temperature. It means that cylindrical wall cooling process takes place. The test problem includes the non-stationary one-dimensional heat conductivity equation. III type boundary conditions are used for the cylindrical wall outer boundaries temperature determination. The medium and material thermal physical properties are taken constant and they are known. The data are given in Table 1.

\section{Mathematical problem statement}

The hollow cylinder temperature distribution has been analyzed by using the following differential equation, given below. $C_{p}$ is a steel heat capacity. The benchmark unit of a heat capacity is a joule 


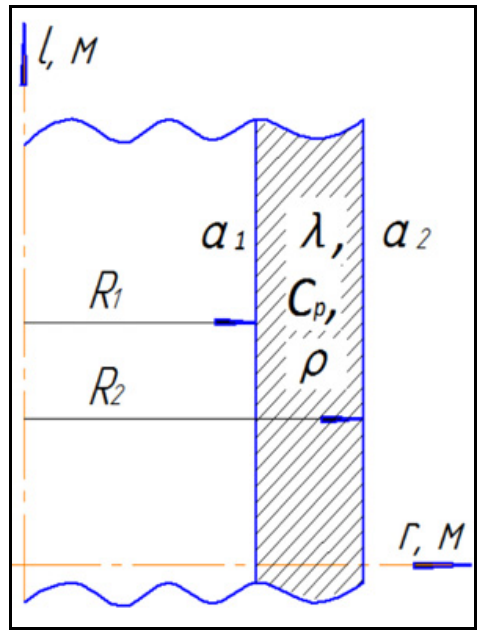

Figure 1. Investigation object diagram.

Table 1.

\begin{tabular}{|l|l|l|}
\hline Symbol & Characteristic & Value \\
\hline$r_{1}$ & Cylindrical wall inner radius & $0,014 \mathrm{M}$ \\
\hline$r_{2}$ & Cylindrical wall outer radius & $0,019 \mathrm{M}$ \\
\hline$C_{p}$ & Cylindrical wall heat capacity (steel 20) & $504,8 Д$ Д $/\left(\mathrm{K} \Gamma \cdot{ }^{\circ} \mathrm{C}\right)$ \\
\hline$\rho$ & Cylindrical wall density (steel 20) & $7800 \mathrm{K \Gamma} / \mathrm{M}^{3}$ \\
\hline$\lambda$ & Cylindrical wall heat conductivity coefficient (steel 20) & $14,278 \mathrm{~B}_{\mathrm{T}} /\left(\mathrm{M} \cdot{ }^{\circ} \mathrm{C}\right)$ \\
\hline$\alpha_{1}$ & Heat-transfer coefficient from the cylindrical wall inner surface to the steam & $1200 \mathrm{~B}_{\mathrm{T}} /\left(\mathrm{M}^{2} \cdot{ }^{\circ} \mathrm{C}\right)$ \\
\hline$\alpha_{2}$ & Heat-transfer coefficient from the smoke to the cylindrical wall outer surface & $60 \mathrm{~B}_{\mathrm{T}} /\left(\mathrm{M}^{2} \cdot{ }^{\circ} \mathrm{C}\right)$ \\
\hline$t$ & Heat time & $170 \mathrm{c}$ \\
\hline$T_{c}$ & Medium temperature & $750{ }^{\circ} \mathrm{C}$ \\
\hline$T_{n}$ & Starting temperature distribution in the wall & $300{ }^{\circ} \mathrm{C}$ \\
\hline
\end{tabular}

per a kilogram per the Celsius degree. $\rho$ is a steel density, measured by kilograms per a cubic meter. $\lambda$ is a steel heat conductivity coefficient, measured in watt per a meter per Celsius degree. $\alpha_{1}, \alpha_{2}$ are the heat-transfer coefficients of the inner surface to the steam and from the smoke to the outer surface respectively, measured in watt per a square meter per Celsius degree. $T_{c}$ is a medium temperature on the boundaries, measured in Celsius degrees.

$$
\begin{gathered}
C_{p} \rho \frac{\partial T(\tau, r)}{\partial \tau}=\lambda\left(\frac{\partial^{2} T(\tau, r)}{\partial r^{2}}+\frac{1}{r} \frac{\partial T(\tau, r)}{\partial r}\right), \quad \tau>0, \quad r_{1}<r<r_{2}, \\
\tau=0 ; \quad T(0, r)=T_{H} \\
r=r_{1} ; \lambda \frac{\partial T\left(\tau, r_{1}\right)}{\partial r}=-\alpha_{1}\left[T_{C}-T\left(\tau, r_{1}\right)\right] \\
r=r_{2} ; \lambda \frac{\partial T\left(\tau, r_{2}\right)}{\partial r}=\alpha_{2}\left[T_{C}-T\left(\tau, r_{2}\right)\right]
\end{gathered}
$$




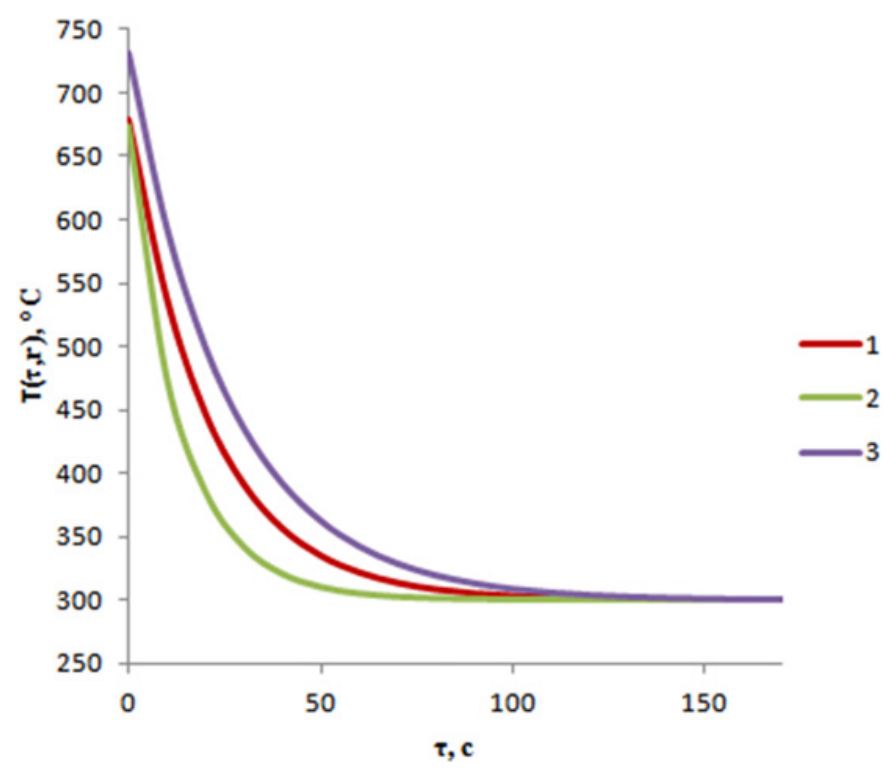

Figure 2. Hollow cylinder inner surface temperature distribution, obtained by usage: 1 - numerical modeling method; 2 - analytical method; 3 - approximate analytical method.

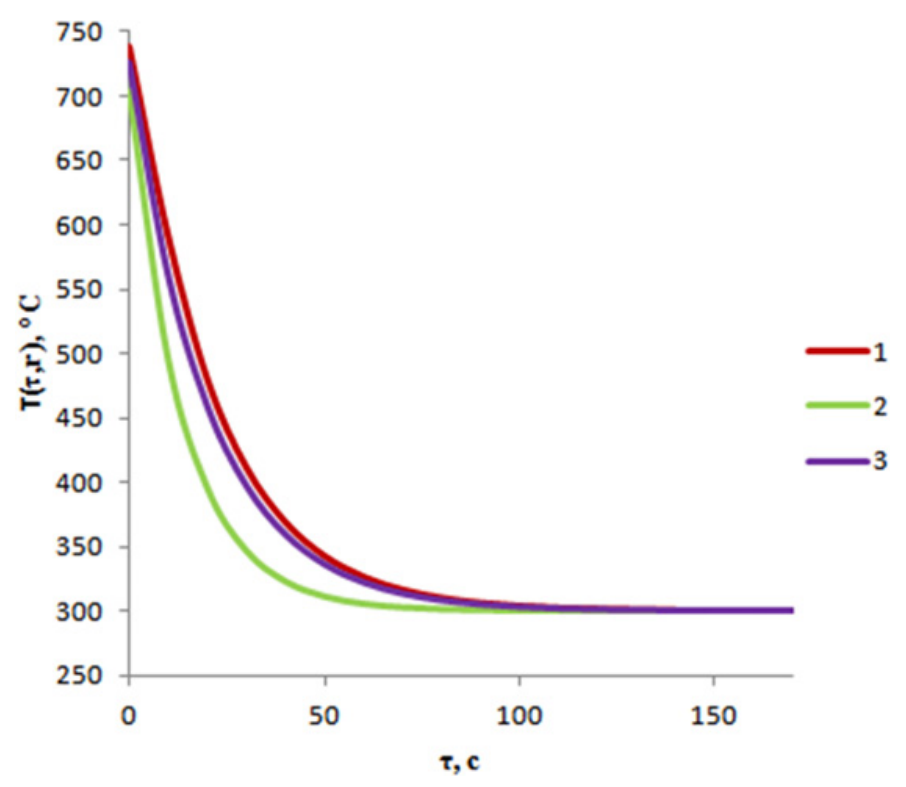

Figure 3. Hollow cylinder outer surface temperature distribution, obtained by usage: 1 - numerical modeling method; 2 - analytical method; 3 - approximate analytical method.

\section{Investigation method}

The above-given mathematical model (1) is determined by the implicit scheme usage as to sweep method $[15,16]$. It's necessary to note, that this solution method isn't only stable to a rounding error, but also 


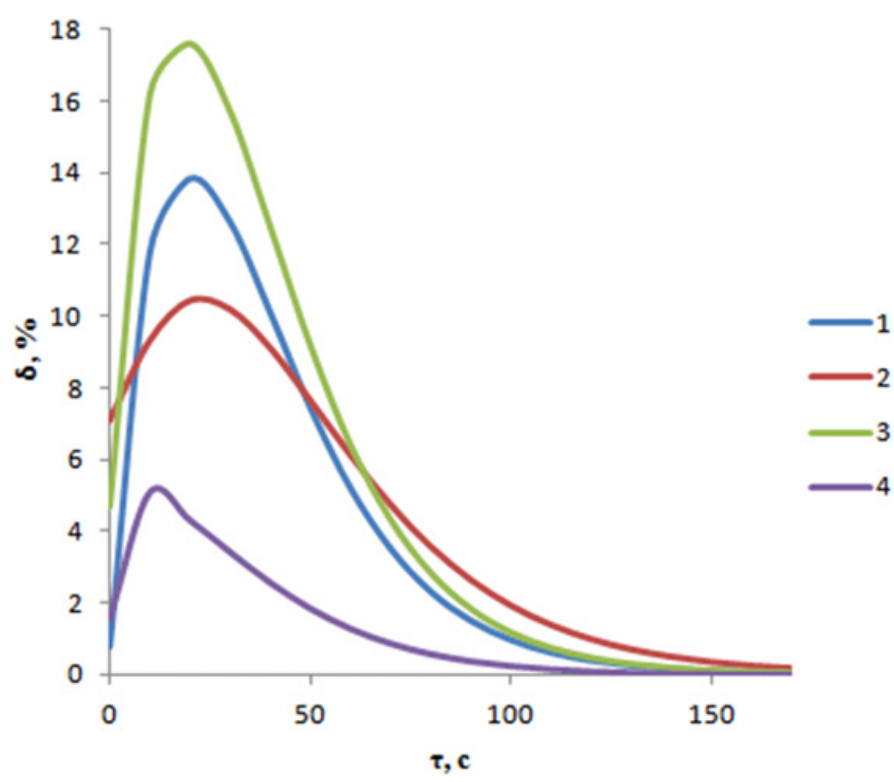

Figure 4. Errors field: 1, 2 - for hollow cylinder inner surface temperature distributions: 1 - comparing with computation results as to analytical method, 2 - approximate analytical method; 3, 4 - for hollow cylinder inner surface temperature distributions: 3 - comparing with computation results as to analytical method, 4 - approximate analytical method.

allows to solve the equations system, having some hundred unknowns. The solution simplicity, made at the computer, is also an advantage.

The problem analytical solution is known [14]:

$$
T(\tau, r)=T_{C}-\sum_{n=1}^{\infty} E_{n} \exp \left(-\mu_{1} \mathrm{Fo}\right) W_{0}\left(\frac{\mu_{n}}{r_{1}}, r\right),
$$

where $\mu_{n}$ are characteristic equation roots:

$$
\begin{gathered}
{\left[\frac{\alpha_{1}}{\lambda} J_{0}(\mu)+\frac{\mu}{r_{1}} J_{1}(\mu)\right]\left[\frac{\alpha_{2}}{\lambda} Y_{0}\left(\mu \frac{r_{2}}{r_{1}}\right)+\frac{\mu}{r_{1}} Y_{1}\left(\mu \frac{r_{2}}{r_{1}}\right)\right]} \\
-\left[\frac{\alpha_{2}}{\lambda} J_{0}\left(\mu r_{1}\right)-\frac{\mu}{r_{1}} J_{1}\left(\mu \frac{r_{2}}{r_{1}}\right)\right]\left[\frac{\alpha_{1}}{\lambda} Y_{0}(\mu)+\frac{\mu}{r_{1}} Y_{1}(\mu)\right]=0, \\
W_{0}\left(\frac{\mu_{n}}{r_{1}}, r\right)=-\left[\frac{\alpha_{1}}{\lambda} Y_{0}\left(\mu_{n}\right)+\frac{\mu_{n}}{r_{1}} Y_{1}\left(\mu_{n}\right)\right] J_{0}\left(\mu_{n} \frac{r}{r_{1}}\right)+\left[\frac{\mu_{n}}{r_{1}} J_{1}\left(\mu_{n}\right)+\frac{\alpha_{1}}{\lambda} J_{0}\left(\mu_{n}\right)\right] Y_{0}\left(\mu_{n} \frac{r}{r_{1}}\right), \\
E_{n}=\frac{\pi^{2}\left(\mu_{n} / r_{1}\right)^{2}}{2}\left[\frac{\alpha_{2}}{\lambda} J_{0}\left(\mu_{n} \frac{r_{2}}{r_{1}}\right)+\frac{\mu_{n}}{r_{1}} J_{1}\left(\mu_{n} \frac{r_{2}}{r_{1}}\right)\right]^{2} \cdot \int_{r_{1}}^{r_{2}} r\left(T_{C}-T_{H}\right) \cdot W_{0}\left(\frac{\mu_{n}}{r_{1}}, r\right)
\end{gathered}
$$




$$
\begin{aligned}
& \times\left\{\left[\left(\frac{\mu_{n}}{r_{1}}\right)^{2}+\left(\frac{\alpha_{2}}{\lambda}\right)^{2}\right)\left[\frac{\alpha_{1}}{\lambda} J_{0}\left(\mu_{n}\right)+\frac{\mu_{n}}{r_{1}} J_{1}\left(\mu_{n}\right)\right]^{2}\right. \\
& \left.\left.-\left(\left(\frac{\mu_{n}}{r_{1}}\right)^{2}+\left(\frac{\alpha_{1}}{\lambda}\right)^{2}\right)\left[\frac{\alpha_{2}}{\lambda} J_{0}\left(\mu_{n} \frac{r_{2}}{r_{1}}\right)+\frac{\mu_{n}}{r_{1}} J_{1}\left(\mu_{n} \frac{r_{2}}{r_{1}}\right)\right]^{2}\right]\right\} .
\end{aligned}
$$

The approximate analytical solution is considered to be as a function, given below. $C_{1}(\tau), C_{2}(\tau), C_{3}(\tau)$ are determined from the starting and boundary conditions in this function.

$$
\Phi(\tau, r)=T_{C}-T(\tau, r)=C_{1}(\tau)+\frac{r}{r_{2}} C_{2}(\tau)+\left(\frac{r}{r_{2}}\right)^{2} C_{3}(\tau),
$$

The following expression, determining hollow cylinder temperature field, was obtained, as a result simple arithmetic operations.

$$
T(\tau, r)=T_{C}\left(1-\exp (\tau L(r))+T_{H} \exp (\tau L(r)\right.
$$

where

$$
\begin{gathered}
L(r)=\frac{\lambda C_{p} \rho\left[\frac{4}{r_{2}^{2}}-\frac{M}{r \cdot r_{2}}\right]}{\left[N-\left(\frac{M \cdot r}{R_{2}}\right)+\left(\frac{r}{R_{2}}\right)^{2}\right]}, \\
M=\frac{\left[\frac{2 \lambda \alpha_{2} r_{1}}{r_{2}^{2}}-\alpha_{1} \alpha_{2}\left(\frac{r_{1}}{r_{2}}\right)^{2}+\frac{2 \lambda \alpha_{1}}{r_{2}}+\alpha_{1} \alpha_{2}\right]}{\left[\frac{\lambda \alpha_{2}}{r_{2}}-\alpha_{1} \alpha_{2} \frac{r_{1}}{r_{2}}+\frac{\lambda \alpha_{1}}{r_{2}}+\alpha_{1} \alpha_{2}\right]}, N=\frac{\left[-\left(\frac{M}{r_{2}}\right)+\frac{M \alpha_{1} r_{1}}{\lambda r_{2}}+\frac{2 r_{1}}{r_{2}^{2}}-\frac{\alpha_{1}}{\lambda}\left(\frac{r_{1}}{r_{2}}\right)^{2}\right]}{\frac{\alpha_{1}}{\lambda}} .
\end{gathered}
$$

Turbo Pascal and MathCAD program environment are used to solve the given problem.

\section{Results and discussion}

The following results have been obtained during the physics-mathematical problem statement solution:

Apparently both the hollow cylinder outer and inner surfaces temperatures can dramatically reduce within temperature at the range $0-60 \mathrm{~s}$ in all three cases as to the obtained results. This law is caused by higher heat transfer coefficients on the appropriate surfaces and high cylindrical wall temperature. The further temperature decreasing process becomes slower and the stationary regime occurs at the time $\tau=170 \mathrm{~s}$, due to the constant thermal physic properties. It's easy to see that the numeric computation results are almost similar to the results, obtained by using the analytical and approximate analytical methods for $F_{o}$ numbers $>0,5$. At the same time the computation results error isn't more than $2 \%$. As to these hypotheses, we can say that the sweep method is applicable for the non-stationary onedimensional differential heat conductivity equations solution for the large $F_{o}$ numbers.

\section{Conclusion}

The sweep method applicability for the non-stationary one-dimensional heat conductivity equations solution has been proved by comparing the test problem numerical results with the results, obtained during its solution by using the analytical and approximate analytical methods in this work. This numeric 
modeling method is applicable for the problems solution similar to the test problem for the large $F_{o}$ number $\left(F_{o}>0,5\right)$.

The reported study was supported by the State Contract 14.740.11.0101.

\section{References}

[1] V.A. Bogachev, O.Ye. Taran, Electric power stations 2, 21-24 (2002)

[2] V.A. Bogachev Electric power stations 5, 20-23 (2009)

[3] G.Ye. Verhovskiy, P.A. Lipaev Power engineering specialist 1, 25-28 (2010)

[4] A.A. Zackirova, R.G. Zaripova, V.I. Semenov Mechanical engineering. Materials technology and metals thermal treatment 2, 123-130 (2008)

[5] V.V. Mulchin, D.S. Lomonosov Mechanical engineering 4, 224-228 (2007)

[6] O.Yu. Semenova Mechanical engineering and power engineering 1, 86-92 (2011)

[7] M.M. Zackirinichnaya, I.R. Kuzeev, V.K. Berdin Oil and gas business 1, 207-214 (2006)

[8] V.V. Artamonov, Control. Diagnostics 1, 8-11 (2010)

[9] V.V. Artamonov, Control. Diagnostics 9, 55-61 (2007)

[10] V.A. Bogachev, T.P. Pshechenckova Power engineering specialist 10, 35-38 (2012)

[11] S.K. Sobolev Differencialniye uravneniya (Differential equations) (N.E. Bauman MSTU, Moscow, 2008)

[12] E.A. Krasnosheckov, A.S. Suckomel Zadachnick po teploperedache (Heat transfer problems book) (Energy, Moscow, 1980)

[13] A.V. Lickov Teoriya teploprovodnosti (Heat conductivity) (Government publishing house of technical theoretical literature, Moscow, 1967)

[14] V.S. Loginov Priblijenniye metodi teplovogo rascheta acktivnih elementov elecktrofizichesckih ustanovock (electro physical equipments active elements thermal calculation approximate methods) (Scientific technical literature publishing, Tomsk, 2000)

[15] A.R. Dorohov, A.S. Zavorin, A.M. Kazanov, V.S. Loginov, Modelirovanie teplovidelyayushih sistem (Modelling of heat-generating complexes) (Publishing house of scientific technical literature, Tomsk, 2000)

[16] Mitchell A.R. Griffiths D.F. The Finite Difference Method in Partial Differential Equations. Wiley, 1980. - 267 p. 